\title{
Anti-DR5 Agonistic Monoclonal Antibody INBRX-109
}

National Cancer Institute

\section{Source}

National Cancer Institute. Anti-DR5 Agonistic Monoclonal Antibody INBRX-109. NCI

Thesaurus. Code C157062.

A recombinant, humanized, agonistic, tetravalent monoclonal antibody directed against human death receptor type 5 (DR5), also called tumor necrosis factor (TNF)-related apoptosis-inducing ligand (TRAIL) receptor 2 (TRAILR2), with potential pro-apoptotic and antineoplastic activities. Upon administration, INBRX-109 specifically binds to exactly four DR5 receptors per molecule, which mimics the interaction of DR5 with its natural ligand TRAIL. This activates DR5 and the death receptor signaling pathway, which results in the activation of caspase cascades, the induction of tumor cell apoptosis and a reduction in proliferation of DR5-expressing tumor cells. Utilizing a tetravalent monoclonal antibody may overcome the challenge of generating effective DR5 clustering while avoiding toxicities associated with anti-drug antibody (ADA) hyper-clustering. DR5, a member of the TNF receptor superfamily (TNFRSF), is expressed on the surfaces of a variety of tumor cells and plays a key role in the induction of tumor cell apoptosis. 